\title{
Stimulus-Specific Adaptation in Auditory Cortex Is an NMDA-Independent Process Distinct from the Sensory Novelty Encoded by the Mismatch Negativity
}

\author{
Brandon J. Farley, Michael C. Quirk, James J. Doherty, and Edward P. Christian \\ Department of Neuroscience, AstraZeneca Pharmaceuticals LP, Wilmington, Delaware 19850
}

\begin{abstract}
The significance of the mismatch negativity (MMN), an event-related potential measured in humans which indexes novelty in the auditory environment, has motivated a search for a cellular correlate of this process. A leading candidate is stimulus-specific adaptation (SSA) in auditory cortex units, which shares several characteristics with the MMN. Whether auditory cortex responses encode sensory novelty, a defining property of the MMN, however, has not been resolved. To evaluate this key issue, we used several variations of the auditory oddball paradigm from the human literature and examined psychophysical and pharmacological properties of multiunit activity in the auditory cortex of awake rodents. We found converging evidence dissociating SSA from sensory novelty and the MMN. First, during an oddball paradigm with frequency deviants, neuronal responses showed clear SSA but failed to encode novelty in a manner analogous to the human MMN. Second, oddball paradigms using intensity or duration deviants revealed a pattern of unit responses that showed sensory adaptation, but again without any measurable novelty correlates aligning to the human MMN. Finally NMDA antagonists, which are known to disrupt the MMN, suppressed the magnitude of multiunit responses in a nonspecific manner, leaving the process of SSA intact. Together, our results suggest that auditory novelty detection as indexed by the MMN is dissociable from SSA at the level of activity encoded by auditory cortex neurons. Further, the NMDA sensitivity reported for the MMN, which models the disruption of MMN observed in schizophrenia, may occur at a mechanistic locus outside of SSA.
\end{abstract}

\section{Introduction}

The role of primary auditory cortex in sensory and cognitive processing is debated. Human and animal studies are both instrumental in defining this role. The view that auditory cortex plays a role in auditory novelty detection arose partially from the discovery of the "mismatch negativity" (MMN), a human scalp recorded event related potential (ERP) component elicited by a sound which deviates from a repeating pattern of recent sounds, and thought to be generated by a temporo-prefrontal network including auditory cortex (Näätänen et al., 2007). Supporting this view, the firing patterns of auditory cortex neurons in animals have been shown to share key properties with the MMN, and have been proposed to represent a unit correlate in circuitry generating the MMN (Ulanovsky et al., 2003; Nelken and Ulanovsky, 2007; von der Behrens et al., 2009). This claim implies that auditory cortex units themselves play an integral role in novelty detection as indexed by the MMN. Critically however, key properties of the human MMN, in particular those relating it to sensory novelty, have not yet been established directly for these units.

\footnotetext{
Received May 21, 2010; revised Sept. 2, 2010; accepted 0ct. 10, 2010.

We are grateful to Anthony Zador, Lung-Hao Thai, Nicolas Catz, David Gurley, and Amy Medd for technical assistance; and to Arnaud Norena and Daniel Mathalon for helpful discussions and comments on the manuscript.

Correspondence should be addressed to Brandon J. Farley, at his present address: Integrative and Adaptive Neurosciences Laboratory, CNRS, University of Provence, 13331 Marseille, France. E-mail: brandon. farley@univ-provence.fr.

DOI:10.1523/JNEUROSCI.2793-10.2010

Copyright $\odot 2010$ the authors $\quad 0270-6474 / 10 / 3016475-10 \$ 15.00 / 0$
}

Two general mechanistic models have been proposed to account for the properties of the MMN. One posits that the MMN arises via a stimulus-specific adaptation (SSA) process whereby feature-specific neural elements are progressively adapted by a repeated stimulus, but a subsequent novel stimulus produces a large activation of nonadapted elements (Jääskeläinen et al., 2004; May and Tiitinen, 2010). Based on this model, the demonstration of SSA alone in auditory signals would be compelling evidence for a correlate of the MMN. Indeed, unit signals recorded from auditory cortex show a greater response to a deviant than a standard stimulus in the oddball paradigm and thus show robust SSA, in both anesthetized and awake animals (Ulanovsky et al., 2003; Nelken and Ulanovsky, 2007; von der Behrens et al., 2009).

However, even though SSA can account for part of the human MMN wave, it has been argued that an additional mechanism (and a different model) is required to explain all its properties, which have been revealed by many variations of the oddball paradigm. For example, the MMN is larger in response to novel than to non-novel stimuli, even when both are presented in contexts that generate the same level of SSA, indicating that some of the signal encodes novelty per se (Schröger and Wolff, 1996; Jacobsen and Schröger, 2001, 2003). These and other data have been suggested to distinguish the MMN as manifesting a memorycomparison mechanism, and to support an alternative model whereby the MMN is produced when a novel event violates a stored neural representation of regularity inherent in the recent sensory environment (Näätänen et al., 2005). In animal studies 
by contrast, these paradigms appropriate to determining whether signals encode novelty per se, in addition to SSA, have not yet been thoroughly tested.

Further, an important piece of mechanistic information about the MMN derives from the observation that it is diminished in schizophrenia (Michie et al., 2000; Umbricht and Krljes, 2005) and that this disruption can be mimicked by NMDA antagonist treatment in healthy volunteers (Umbricht et al., 2000). Whether SSA in auditory cortex units is similarly sensitive to NMDA antagonists is untested, however. To further understand the role of auditory cortex units in sensory novelty processing and their relation to the MMN, we systematically distinguished SSA vs novelty components in unit signals as well as tested their NMDA dependence. Recordings were performed in chronically implanted freely behaving rats so that physiological and pharmacological properties would be not be confounded by effects of anesthesia.

\section{Materials and Methods}

Surgical procedure. Research procedures performed on animals were approved by the AstraZeneca Institutional Animal Care and Use Committee in accordance with regulations established by AAALAC. Animals were anesthetized with $1.5 \%$ gaseous isoflurane $/ \mathrm{O}_{2}$ mixture. Body temperature was maintained at $37^{\circ}$ with a homeothermic blanket. A craniotomy and durotomy were performed just large enough to allow implantation of a 12 electrode array (2 rows of six insulated steel wires; diameter, 50 $\mu \mathrm{m}$; center-to-center spacing, $400 \mu \mathrm{m}$ ) into primary auditory cortex (coordinates relative to bregma: anteroposterior, $-4.3 \mathrm{~mm}$; mediolateral, $6.5 \mathrm{~mm}$; depth, $4.5 \mathrm{~mm}$ ). A nano-connector strip (Omnetics Connector Corp.) holding the electrodes was permanently anchored to the skull with dental acrylic. Animals recovered $>10 \mathrm{~d}$ before studies. To verify the location of electrode tips in auditory cortex, a small marking lesion was made with DC current, and tissue was processed for Nissl staining (Fig. 1).

Acoustic stimuli and stimulation paradigms. Recordings were made while the animal freely explored a Plexiglas chamber 30 (length) $\times 21$ (width) $\times 33$ (height) $\mathrm{cm}$ housed in a double-walled acoustic box. Pure tone stimuli were produced by a Tucker-Davis Technologies model RP2 Audio Generator and played from a free-field speaker (Tucker-Davis Technologies, model FF1) centered on the ceiling panel of the recording chamber. Sound stimulus intensity was calibrated by placing a microphone (Bruel and Kjaer, model 4939) in multiple positions near the bottom of the chamber at the estimated head height of a rat during walking or quiescence. After averaging over multiple positions we noted a linear falloff of intensity with increasing frequency. The generator output was programmed to compensate for this. Adequate control of positional variations in intensity within the chamber was confirmed by the finding that frequency-level tuning properties of multiunit responses accorded well with those extensively documented (Fig. 1).

To characterize frequency-level tuning of each multiunit cluster we presented a matrix of pure-tones (duration: $100 \mathrm{~ms}$; interstimulus interval [ISI]: $600 \mathrm{~ms}$ ) pseudo-randomly at one of 10 frequencies (spaced by $1 / 2$ octave) and one of 7 intensities (spaced by $10 \mathrm{~dB}$ ). The entire session,
B

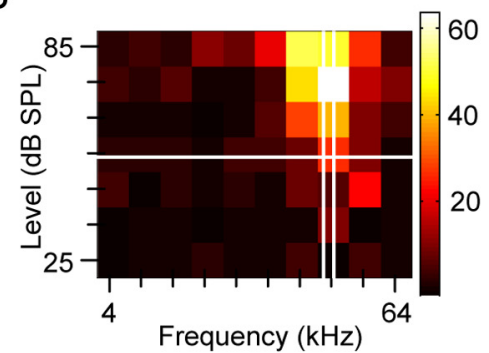

D
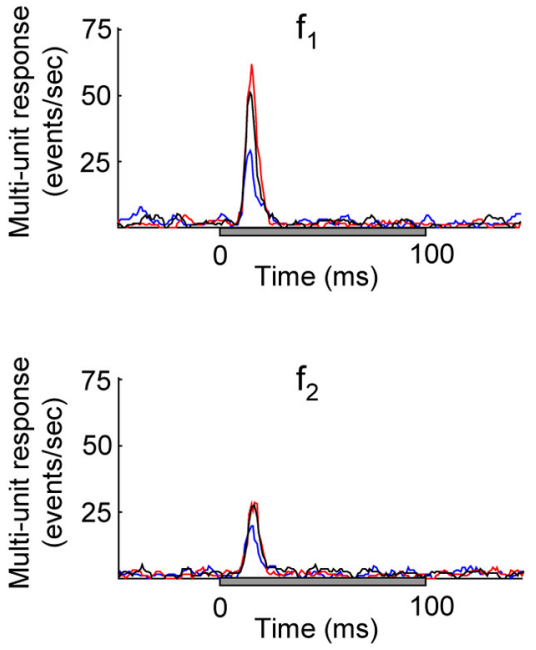

Figure 1. Frequency-level tuning and stimulus-specific adaptation from primary auditory cortex multiunit site in awake rat. All data in this figure are obtained from a single recording site. $A$, Nissl-stained coronal slice of rat brain containing electrolytic lesion

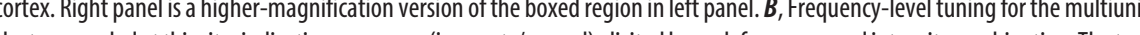
列 (probability of occurring $p=1 / 9)$ in oddball block 2 ; whereas the $f_{2}(34 \mathrm{kHz}, 50 \mathrm{~dB}$, in this a low probably $(p=1 / 9)$ in the context of nine different tones, all occurring with $p=1 / 9$, where adjacent frequencies are separated by $\Delta f=0.1$. $D$, Multiunit PSTHs showing the responses for this example site during the frequency oddball paradigm to the $f_{1}$ or the $f_{2}$ stimulus occurring as a standard (blue), deviant (red), or control (black).

which spanned $70 \mathrm{~min}$, encompassed 100 repeats of each tone. The average response to the 100 tone repeats was calculated. The characteristic frequency was defined as the frequency at which a significant multiunit response was elicited at the lowest sound amplitude, which was defined as the intensity threshold.

Frequency, intensity, and duration oddball paradigms were all evaluated over the course of the study. For the frequency oddball paradigm (Fig. 1), we presented two pure-tone stimuli $f_{1}$ and $f_{2}$, separated in frequency by $\Delta f=0.1$, where $\Delta f=\left(f_{1}-f_{2}\right) /\left(f_{1} \times f_{2}\right)^{1 / 2}$. This $\Delta f$ corresponds to $\sim 1 / 10$ octave. The two frequencies were centered on the characteristic frequency of the multiunit response for the recording site. The intensity of each stimulus was $20 \mathrm{~dB}$ above the threshold and the duration was $100 \mathrm{~ms}$. Three types of "blocks" were presented with each block containing 300 sequential stimuli (ISI $600 \mathrm{~ms}$ ). In the first block type, the $f_{1}$ stimulus was the "standard" in an oddball paradigm, occurring with a probability of $8 / 9(\sim 89 \%)$ and the $f_{2}$ stimulus was the "deviant," occurring with a probability of $1 / 9(\sim 11 \%)$. In the second block type, probabilities were reversed in the oddball paradigm such that the $f_{1}$ became the deviant and the $f_{2}$ the standard. In the third "control" block 9 different tones with equally spaced $(\Delta f=0.1)$ frequencies, including $f_{1}$ and $f_{2}$ in the center of the frequency range, were presented, each with an equal probability (1/9). Thus, the probability of the $f_{1}$ or $f_{2}$ stimulus 
occurring in the control block (and thus the average ISI) was the same as in the deviant condition, but the context of the surrounding tones was different. The $f_{1}$ or $f_{2}$ stimulus was considered rare and novel when serving as a deviant in the oddball paradigm (i.e., occurring infrequently in the context of one commonly presented standard tone), whereas the $f_{1}$ or $f_{2}$ was rare, but not novel in the control block (i.e., occurring in the context of several other equally uncommon tones). Each of the three block types was presented 10 times in random order, with $60 \mathrm{~s}$ in between each block. Alternating between block types provided a randomization strategy aimed at mitigating possible effects on auditory responses of ongoing changes in the vigilance state of an animal over the $2 \mathrm{~h}$ time course of the oddball paradigm.

For the intensity oddball paradigm (see Fig. 3), the intensity of the stimulus was either $15 \mathrm{~dB}\left(i_{1}\right)$ or $25 \mathrm{~dB}\left(i_{2}\right)$ above threshold, the frequency was the characteristic frequency and the duration was $100 \mathrm{~ms}$. The firing rate-intensity functions for each of the multiunits included in the intensity oddball study were monotonic within the intensity ranges examined, although it is possible that non-monotonic properties might have been revealed at higher intensities (supplemental Fig. 1, available at www.jneurosci.org as supplemental material). For the duration oddball paradigm (see Fig. 3), the duration of the stimulus was either $50 \mathrm{~ms}\left(d_{1}\right)$ or $125 \mathrm{~ms}\left(d_{2}\right)$, the frequency was the characteristic frequency and the intensity $20 \mathrm{~dB}$ above threshold. For the intensity (and duration) oddball paradigms, two block types were interleaved, the first in which the $i_{1}\left(d_{1}\right)$ stimulus was the standard (i.e., $89 \%$ occurrence) and the $i_{2}\left(d_{2}\right)$ was the deviant, and the second in which their contextual identities were reversed.

For pharmacological experiments, which were performed using the frequency oddball paradigm, subcutaneous drug injections were administered immediately after completing the entire paradigm. Then, $10 \mathrm{~min}$ after injection of either a control solution $(0.9 \% \mathrm{NaCl}), 0.03 \mathrm{mg} / \mathrm{kg}$ MK801 (a noncompetitive NMDA antagonist), or $0.1 \mathrm{mg} / \mathrm{kg}$ MK801, the entire paradigm was repeated.

In vivo electrophysiology and data analysis. The electrode connector implanted on the skull was mated with a 16-channel headstage preamplifier (HS-18; Neuralynx) attached to a flexible tether and commutator, allowing unimpeded movement of the animal during the recording. The signal from each electrode was split into a continuously sampled channel for recording local field potential (LFP) signals and a spike channel for unit signals. Filtering and digitization were performed with a Digital Lynx 64 system (Neuralynx).

The LFP signal was filtered (low-pass, $1 \mathrm{~Hz}$; high-pass, $475 \mathrm{~Hz}$ ) and digitized at $2 \mathrm{kHz}$. LFP responses were only analyzed in the oddball paradigm from those electrodes which also had responsive multiunits. In $>90 \%$ of these sites, the sound-evoked ERP from the LFP showed a prominent initial negativity with latency $\sim 20 \mathrm{~ms}$, which we term the N20. A negativity of this latency is consistent with what has been shown previously from the middle layers of the rat auditory cortex (Barth and Di, 1990; Szymanski et al., 2009). Although the average population response from the LFPs also displayed an additional prominent positivity with a latency of $\sim 110 \mathrm{~ms}$, the existence of this component was more variable across recordings, occurring in $\sim 70 \%$ of cases. We analyzed the behavior of both the N20 and P110 components during the frequency oddball paradigm.

The neuronal spike signal was filtered (low-pass, $600 \mathrm{~Hz}$; high-pass, $3000 \mathrm{~Hz}$ ), and events which crossed an arbitrarily set threshold value above the background were digitized over a $3 \mathrm{~ms}$ time window and recorded. The quality of the waveforms and cluster separation from background was continuously monitored online for drift. Following the experiment, recorded waveforms were visualized using NeuroExplorer software (version 4; Plexon) and obvious artifactual events removed. The waveforms from a small percentage of the 40 pure-tone responsive clusters (from 14 animals), from which the data of this study derive, had an autocorrelation where $<5 \%$ of events occurred within $\pm 0.5 \mathrm{~ms}$ and thus were likely single units, but the majority of clusters were considered multiunits based on this criterion.

For quantifying multiunit responses to stimuli during the oddball paradigm, the number of events crossing a threshold during a-specific time epoch was counted. This time epoch was the same for the standard, deviant and control conditions, and ranged from the point when the response to the standard first exceeded the prestimulus baseline by 2 standard deviations to the point at which it returned to this value. For LFPs, we calculated the amplitude of the N20 and P110 components for the standard, deviant, and control conditions. To quantify the response difference between the deviant and standard in the frequency oddball paradigm, we used a normalized stimulus-specific adaptation index (SSAI) for each frequency, as formulated by Ulanovsky et al. (2003) and defined as follows: $\operatorname{SSAI}\left(f_{\mathrm{i}}\right)=\left[d\left(f_{\mathrm{i}}\right)-s\left(f_{\mathrm{i}}\right)\right] /\left[d\left(f_{\mathrm{i}}\right)+s\left(f_{\mathrm{i}}\right)\right](i=1,2)$ or for the average of both stimuli, $\operatorname{SSAI}(f)=\left[d\left(f_{1}\right)+d\left(f_{2}\right)-s\left(f_{1}\right)-s\left(f_{2}\right)\right] /$ $\left[d\left(f_{1}\right)+d\left(f_{2}\right)+s\left(f_{1}\right)+s\left(f_{2}\right)\right]$, where $d\left(f_{\mathrm{i}}\right)$ and $s\left(f_{\mathrm{i}}\right)$ are responses to frequency $f_{\mathrm{i}}$ when it was a standard or a deviant, respectively. Analogous indices were used to compare the responses of the deviant and standard in the intensity and duration paradigms, by substituting in the formulas above the response to frequency $f_{\mathrm{i}}$ with the response to intensity $i_{\mathrm{i}}$ or duration $d_{\mathrm{i}}$, respectively. Additionally, to quantify the degree of "novelty" encoded in the responses in the frequency oddball paradigm, we compared the responses to the deviant and control stimuli, defining a "novelty index" NI $\left(f_{\mathrm{i}}\right)=\left[d\left(f_{\mathrm{i}}\right)-c\left(f_{\mathrm{i}}\right)\right] /\left[d\left(f_{\mathrm{i}}\right)+c\left(f_{\mathrm{i}}\right)\right]$ where $d\left(f_{\mathrm{i}}\right)$ and $c\left(f_{\mathrm{i}}\right)$ are responses to frequency $f_{\mathrm{i}}$ when it was a deviant or a control, respectively.

For multiunit population PSTHs, the PSTH to the standard, deviant, and control (for the $f_{1}$ or $f_{2}$ stimulus) for each multiunit site was normalized to the peak of the respective standard response. These normalized responses subsequently were averaged over all multiunit sites. A similar procedure was used to normalize LFP population ERPs, with responses normalized to the peak of the N20 elicited by the standard. To characterize the kinetics for development of SSA in an individual recording, the responses to the standard or deviant stimuli were combined across all 10 repeats of that block type, based on their position in the oddball sequence (from the first position to the $300^{\text {th }}$ position). Responses were then normalized to the mean value of the preinjection standard response and plotted at their original position in the 300-stimulus-long (i.e., 180-slong) time scale. The time course of the multiunit population standard response, before and following drug injections, was fitted to the following exponential function: decay_size $\times\left(1-e^{-t / \tau}\right)+$ asymptote. Here, $\tau$ represents the time constant of the adaptation (Ulanovsky et al., 2004).

For statistics, the nonparametric sign test was used to compare paired samples. For pharmacology, the oddball paradigm recording for each drug condition was preceded by a preinjection baseline oddball paradigm recording, and the baseline responses were subtracted from the respective drug condition responses. Then, effects across drug conditions were compared using the nonparametric Kruskal-Wallis ANOVA.

\section{Results}

\section{Multiunit responses exhibit SSA, but no novelty profile}

Multiunit activity from electrodes chronically implanted within the auditory cortex in freely moving rats exhibited clear timelocked responses during the presentation of acoustic stimuli (Fig. $1 A$ ). A subset of multiunit clusters showed frequency-level tuned responses to pure-tone stimuli (Hromádka et al., 2008), and we focused on these sites because standard and oddball stimulus frequencies could be presented within a standardized region of the receptive field across multiple recordings. Figure $1 B$ shows the responses to pure-tone stimuli for a multiunit cluster displaying an exemplary "V-shaped" tuning curve at low to moderate sound levels for a limited range of sound frequencies.

After measuring the tuning-curve characteristics of each responsive multiunit site, we presented a frequency "oddball paradigm" (Fig. 1C) using stimuli centered near the characteristic frequency and with moderate intensities (see Materials and Methods). During the ensuing oddball paradigms, the response to the same physical tone was compared in different sensory contexts. First, we compared the response elicited when the tone was either a high-probability "standard" stimulus or a low-probability "deviant" stimulus embedded within a series of identical standards. The responses from the multiunit site exemplified in Figure 1 showed strong sensory context 
A

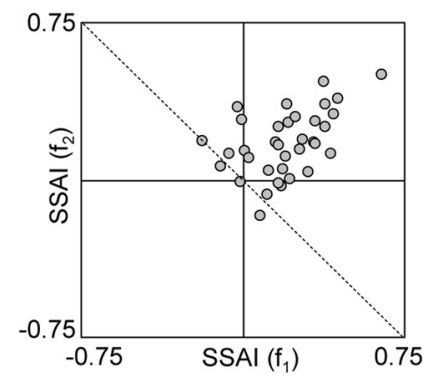

B
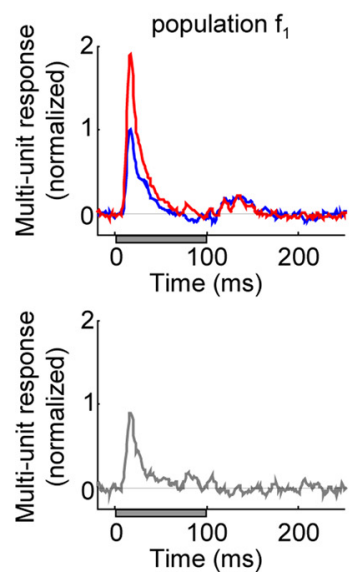
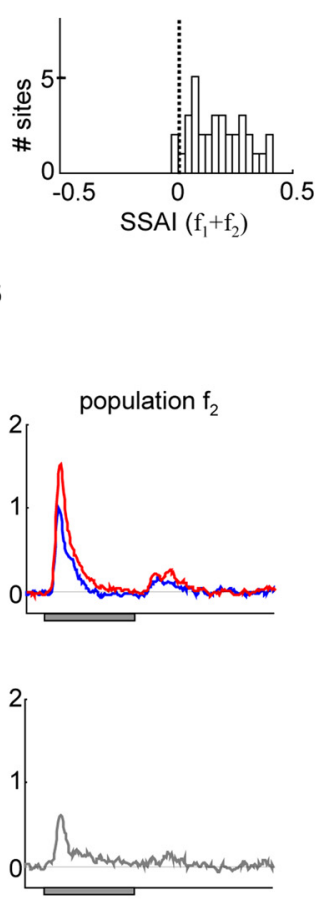

C

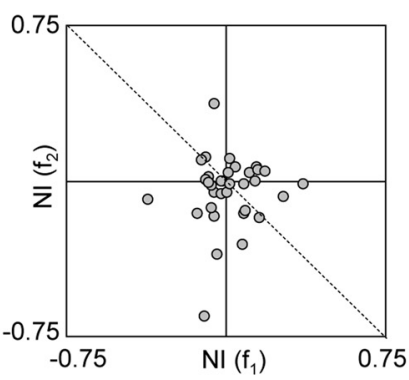

D
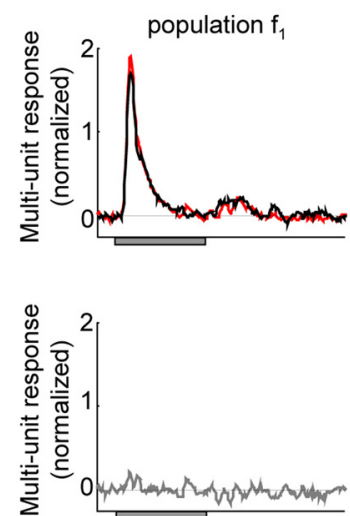
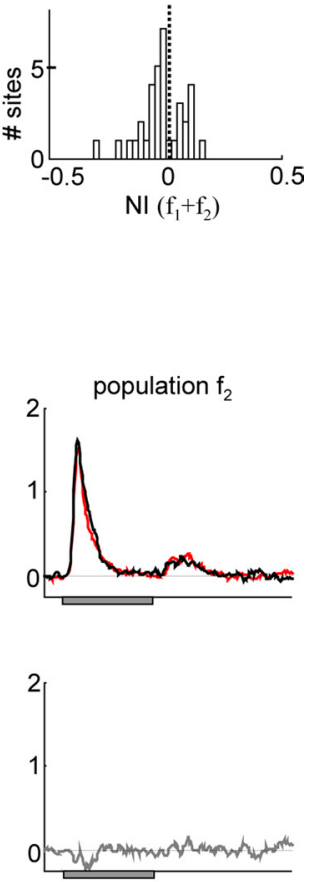

Figure 2. Auditory cortex multiunit population responses during frequency oddball paradigm. A, Stimulus-specific adaptation index for the $f_{1}$ and $f_{2}$ stimuli plotted for each multiunit site. A positive value indicates that the response to the deviant was greater than that to the standard. Histogram represents the averaged $f_{1}$ and $f_{2}$ stimulus-specific adaptation index, calculated for each site and binned. $\boldsymbol{B}$, The population PSTH for the standard (blue), deviant (red), and their difference (gray, below), shown separately for the $f_{1}$ and $f_{2}$ frequencies. $\boldsymbol{C}$, Novelty index for the $f_{1}$ and $f_{2}$ stimuli plotted for each multiunit site. A positive value indicates that the response to the deviant was greater than that to the control. Histogram represents the averaged $f_{1}$ and $f_{2}$ novelty index, binned for all sites. D, The population PSTH for the deviant (red, same as in $\boldsymbol{B}$ ), control (black), and their difference (gray, below). For the population, the response to the deviant was significantly greater than that to the standard but not greater than that to the control, indicating strong evidence for stimulus-specific adaptation (SSA) but no evidence for novelty-encoding.

dependence. Specifically, the response to a tone was stronger when it occurred as a deviant, rather than as a standard (Fig. 1D). This response pattern is consistent with that observed previously in auditory cortex units (Ulanovsky et al., 2003; von der Behrens et al., 2009), and supports the presence of SSA. However, we wished to additionally determine whether the increased response to the deviant could be fully explained by SSA, or if it also encoded a novelty aspect of the stimulus relative to its background context.

To distinguish between these possibilities, in addition to the standard and deviant stimuli, we used a "control" stimulus condition which was pioneered in human studies of the MMN (Fig. $1 C$, bottom) (also see Materials and Methods) (Schröger and Wolff, 1996). In this control condition, the tone was presented at an identical low probability as in the deviant condition, to elicit a similar low-level of adaptation as in the latter. However, unlike the deviant which was embedded in the context of one highprobability standard tone, the control was embedded in a random sequence of eight other tones of differing frequency, all with equally low-probability. Thus, unlike the deviant the control tone was not novel relative to the background sensory context. For the exemplary multiunit site, the response to the novel, deviant tone was not largely different from the response to the non-novel, control tone (Fig. 1D). This indicates that the novelty aspect itself of the deviant tone was not encoded in the multiunit responses, and does not account for the different response levels elicited by standards and deviants in the oddball paradigm. Rather, the results suggest that the different responses observed between con- ditions could be accounted for fully by SSA, and more accurately reflect a relative decrease in the standard as a result of adaptation, compared with the deviant or control for which adaptation is comparatively nominal.

To disambiguate the alternative contributions of SSA and novelty across all multiunit recordings showing frequencylevel tuning, we quantified for each site the response difference elicited by standard and deviant stimuli using the stimulusspecific adaptation index (SSAI; see Materials and Methods). In parallel, we quantified the response difference elicited by deviant and control stimuli using the novelty index (NI; see Materials and Methods). Across the population, the average response to the deviant in the oddball paradigm was significantly greater than that to the standard (Fig. $2 A$ ) for both the $f_{1}$ (Sign test, $p<0.001$ ) and $f_{2}$ (Sign test, $p<0.0001$ ) frequencies, as reflected by the positive SSAIs. This pattern is also clearly apparent in the population PSTH profiles for the standard and the deviant (Fig. $2 B$ ). Thus, multiunit responses in auditory cortex exhibit robust evidence for SSA.

The above observations in isolation are also consistent putatively with a novelty correlate. However, in agreement with the representative experiment in Figure $1 D$, there was no significant difference across all experiments between the response to a given tone in the deviant vs control contexts for either the $f_{1}$ (Sign test, NS) or $f_{2}$ (Sign test, NS) frequency, as reflected by the NI (Fig. $2 C)$. Likewise, there was no difference between the control and deviant population PSTH profiles (Fig. 2D). These summary re- 
sults thus support that SSA alone (without a novelty contribution) accounts for the enhanced response elicited from neurons in the auditory cortex to deviant compared with standard tones in the oddball paradigm.

\section{Intensity- and duration-oddball paradigms elicit adaptation but not novelty responses}

Additional evidence distinguishing between novelty and adaptation contributions to human evoked potentials has come from oddball paradigms in which stimulus dimensions other than frequency are varied. For example, in an oddball paradigm where sound intensity is the variable, the occurrence of a loud deviant tone (in a background of soft tones) or a soft deviant tone (in a background of loud tones) elicits an MMN. This behavior is difficult to explain by an adaptation process, and has been interpreted to suggest that the MMN can encode intensity novelty (Näätänen and Picton, 1987).

We analyzed multiunit responses during an intensity oddball paradigm (Fig. $3 A-D$ ) by applying a protocol analogous to that used in the above frequency oddball studies with tone presentation blocks in which higher ( $25 \mathrm{~dB}$ above threshold) and lower intensity (15 dB above threshold) tones were alternated as frequent standards or infrequent deviants (Fig. 3A). Different trends for the response amplitudes were observed when the data were analyzed separately for soft and loud stimuli presented in standard and deviant contexts (Fig. 3B-D). A representative multiunit response profile in this paradigm is shown in Figure $3 B$. Loud deviants generated significantly greater responses than loud standards (Fig. $3 C, D$; Sign test, $p<0.01$ ). In contrast, no significant response difference, was observed between soft deviants and soft standards (Fig. 3C,D; Sign test, NS). These data are not consistent with the novelty-encoding profile of the human MMN, where both soft and loud stimuli generate greater responses as deviants than as standards.

Auditory oddball paradigms with duration as the variable parameter have also been used in humans to discern a novelty-like response profile in neural signals. We thus used a duration oddball paradigm (Fig. $3 E-H)$ in which shorter $(50 \mathrm{~ms})$ and longer $(125 \mathrm{~ms})$ tones were alternated as frequent standards or infrequent deviants (Fig. $3 E$ ), and analyzed responses in a comparable manner to the other physical dimensions studied above. In this paradigm, a novelty-encoding profile would consist of the responses to (short or long) deviant stimuli being greater than the responses to standard stimuli, but specifically at response latencies $>50 \mathrm{~ms}$, which is the first time point where a difference exists between a deviant and a standard stimulus.

We found that the auditory cortex multiunit responses to duration standards and deviants differed, but not in a manner consistent with a novelty-encoding profile. First, we observed opposite response profiles for short and long sounds when each was presented in a standard vs deviant context. A representative multiunit site is shown in Figure $3 F$. We found that across the population, the peak response to long deviants was significantly greater than that to long standards (Fig. 3G,H; Sign test, $p<$ 0.01 ). On the other hand, the peak response to short deviants was significantly less than that to short standards (Fig. 3G, H; Sign test, $p<0.01)$. These responses do not accord with a novelty profile, which would be distinguished by larger responses to deviants than to standards for both long and short tones. Further, we found no multiunit responses showing significant increases after a latency of $50 \mathrm{~ms}$ (the time at which the deviant diverges physically from the standard) for deviants relative to standards for either short or long tones. Thus no signal in the response profiles
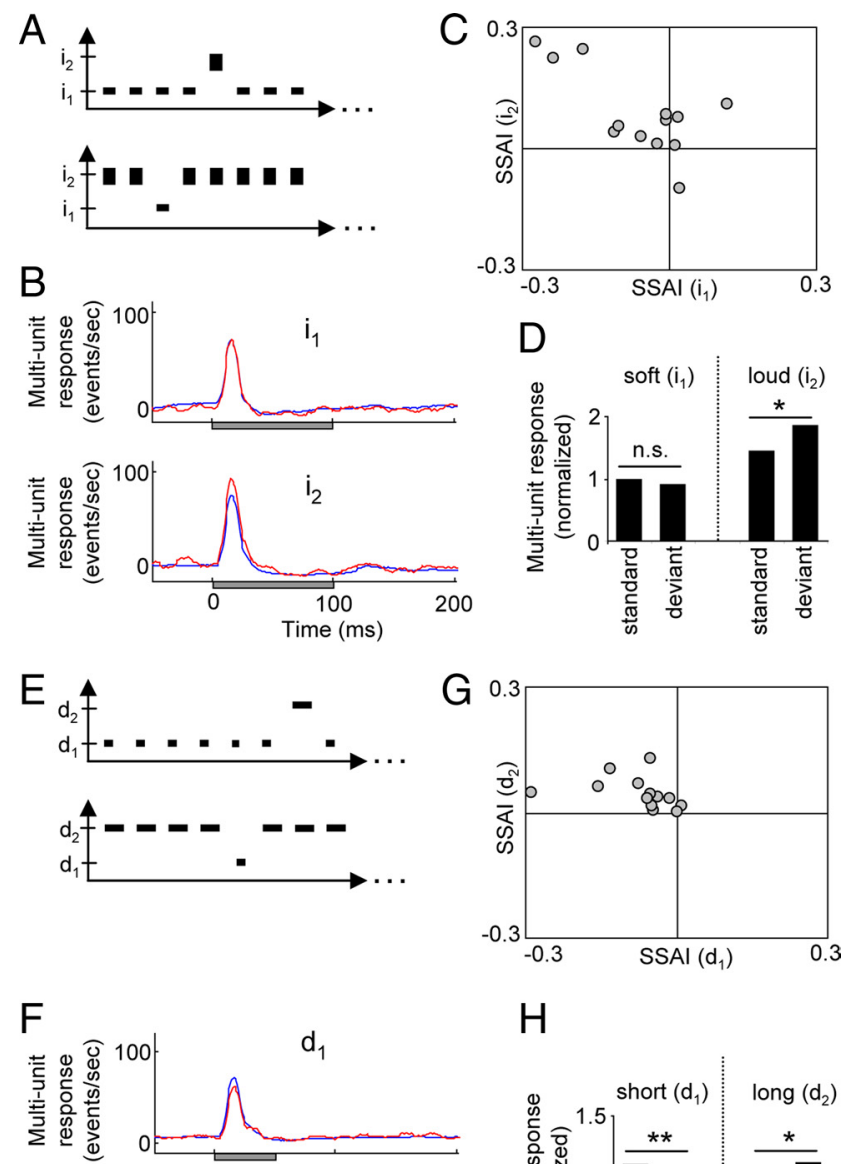

$\mathrm{H}$
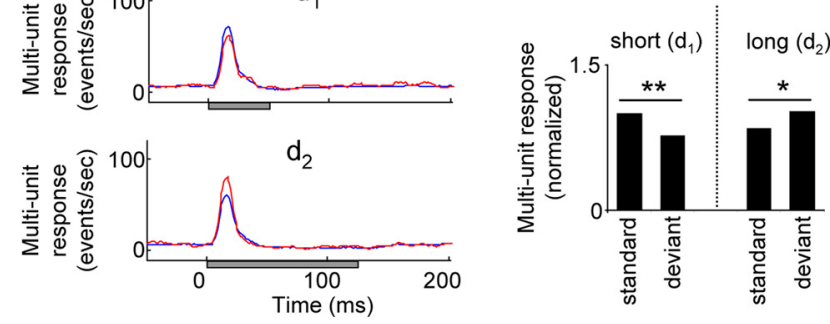

Figure 3. Multiunit population responses during intensity and duration oddball paradigms. $\boldsymbol{A}$, Schematic of the intensity oddball paradigm. The $i_{1}$ stimulus (whose intensity is $15 \mathrm{~dB}$ above threshold) occurs as a "standard" in block 1 and a "deviant" in block 2; whereas the $i_{2}$ stimulus (whose intensity is $25 \mathrm{~dB}$ above threshold) is a "deviant" in block 1 and a "standard" in block 2. $\boldsymbol{B}$, Multiunit PSTH showing the responses for a representative multiunit site during the intensity oddball paradigm. The deviant response (red) for this site is greater than the standard response (blue) for the loud $\left(i_{2}\right)$ stimulus but not for the soft $\left(i_{1}\right)$ stimulus. $C$, Stimulus-specific adaptation index for the $i_{1}$ and $i_{2}$ stimuli plotted for each multiunit site. $D$, The median response magnitude over all multiunit sites for each condition indicated. Note that the response to the loud deviant was greater than that to the loud standard; however, the responses to the soft deviant and standard were not different. $\boldsymbol{E}$, Schematic of the duration oddball paradigm. The $d_{1}$ stimulus (50 ms duration) is a standard in block 1 and a deviant in block 2; the $d_{2}$ stimulus ( 125 ms duration) is a deviant in block 1 and a standard in block 2.F, Multiunit PSTH showing the responses for a representative multiunit during the duration oddball paradigm. The deviant response (red) is greater than the standard stimulus (blue) for the long $\left(d_{2}\right)$ stimulus but smaller for the short $\left(d_{1}\right)$ stimulus. G, Stimulus-specific adaptation index for the $d_{1}$ and $d_{2}$ stimuli plotted for each multiunit site. $\boldsymbol{H}$, The median response magnitude over all multiunit sites for each condition indicated. Note that the response to the long deviant was greater than that to the long standard; however, the responses to the short deviant were less than that to the short standard.

could be associated with auditory novelty when the deviant was presented.

Together, these data indicate that auditory cortex units exhibit SSA, but fail to show a novelty detection component essential to the definition of MMN. Thus the response properties of auditory cortex units in the oddball paradigm are dissociable from those of the MMN, as demonstrated here using multiple auditory stimulus dimensions. 
NMDA independence of SSA

An additional property we tested for SSA encoded by auditory cortex units was its sensitivity to noncompetitive NMDA antagonists. The MMN is disrupted by these agents, but presuming that the MMN as typically measured is comprised of both novelty and adaptation components (Schröger and Wolff, 1996), it is unknown which of these is the substrate of the disruption. Figure 4 shows a typical experiment evaluating the effects of the NMDA antagonist, MK801, at a subanesthetic dose of 0.1 $\mathrm{mg} / \mathrm{kg}$ that is associated with recapitulating schizophrenia-like symptomatology in rodents (Sams-Dodd, 1996). The multiunit response in the frequency oddball paradigm before treatment showed clear SSA, as evidenced by the larger response to the oddball than to the standard tone (Fig. $4 A$, thick lines). MK801 had a strong suppressive effect on evoked multiunit responses (Fig. 4A, thin lines). However, the responses to both the standard and the deviant were suppressed (Fig. 4A), suggesting that the SSA process was not specifically affected.

To explore further the specificity of effect of MK801 on SSA, we analyzed the temporal evolution of the responses to the standard and deviant stimuli during the oddball paradigm. SSA is a dynamic process, whereby responses to a standard and deviant have similar amplitudes early in a train of standards, but diverge during the train due to a progressive decline in the standard response with development of habituation (Ulanovsky et al., 2004). For this representative experiment, following MK801 injection, the initial responses to both the standard and deviant at the beginning of the oddball paradigm were both strongly suppressed compared with in the preinjection period (Fig. 4B). Nevertheless, adaptation with an apparently similar time course still developed for the standard relative to the deviant, suggesting that the dynamic process of SSA remained intact.

This pattern, where MK801 affected the magnitude of auditory cortex multiunit responses but not SSA or its dynamics, was confirmed further by examining the effects of two doses of MK801 on the auditory cortex multiunit responses across multiple experiments (Fig. 5). Vehicle treatment had no effect on the median multiunit response magnitude to the standard or deviant stimulus compared with the preinjection period (Fig. 5A; Sign test, NS for standard or deviant). In contrast, injection of a low $(0.03 \mathrm{mg} / \mathrm{kg})$ dose of MK801 suppressed responses compared with the preinjection period (Fig. $5 A$; sign test, $p<0.01$ for both standard and deviant). Injection of a higher $(0.1 \mathrm{mg} / \mathrm{kg})$ dose of MK801 suppressed even more dramatically the responses to both standard and deviant stimuli (Fig. 5A; sign test, $p<0.01$ and $p<$ 0.05 for standard and deviant, respectively), suggesting a dose dependence for this suppressive effect (Fig. 5A-C). An ANOVA confirmed that the responses to both standards and to deviants were differentially affected between the three conditions of saline, low-dose MK801, or high-dose MK801 injection (Fig. 5B,C; Kruskal-Wallis ANOVA; $p<0.01$ and $p<0.05$ for standard and deviant, respectively). Post hoc comparisons indicated that the differences were accounted for by a significant suppression of both the standard and the deviant response by the high dose of
MK801 relative to saline (Mann-Whitney $U$ test, $p<0.01$, for both the standard and deviant).

These results indicated that NMDA antagonists suppress the responses of auditory cortex units evoked by standard or deviant stimuli in the oddball paradigm; importantly, however, the magnitude of the suppression was not different between the standard and deviant. Because SSA is defined as the difference between the response to the deviant and standard, it follows that MK801 did not affect the magnitude of SSA. Figure $5 D$ substantiates this graphically by demonstrating lack of a MK801-induced systematic change in SSA across the sampled population of multiunits (Sign test, NS for vehicle, low-dose, and high-dose MK801). An ANOVA confirmed no difference between the effects of saline versus the two doses of MK801 on SSA (Fig. 5E; Kruskal-Wallis ANOVA, NS).

To further substantiate the conclusion that NMDA antagonists do not affect SSA, the effects of MK801 were evaluated on the kinetics of SSA development for the multiunit population (Fig. $5 F$ ), corresponding to the analysis shown for the individual sample in Figure $4 B$. The baseline responses to both the standard and deviant stimuli, at the beginning of the oddball paradigm, were suppressed following the MK801 injections relative to the vehicle injection. Nevertheless, a time-(and stimulus-)dependent suppression of the standard response relative to the deviant evolved in time in all three treatment conditions, and with similar kinetics. The mean time constant of adaptation to the standard stimulus (see Materials and Methods) following drug injection was similar in all three conditions $(4.7,4.2$, and $4.2 \mathrm{~s}$ for vehicle, low-dose MK801, and high-dose MK801, respectively).

\section{SSA in local field potentials}

LFP responses from the recordings were also analyzed, based on reasoning that these may more closely correlate with evoked po- 
A

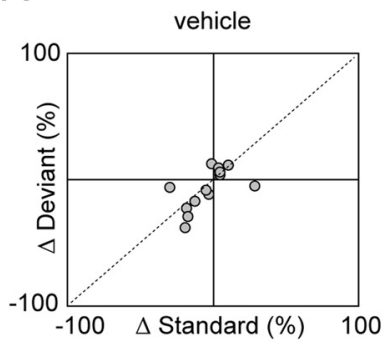

D

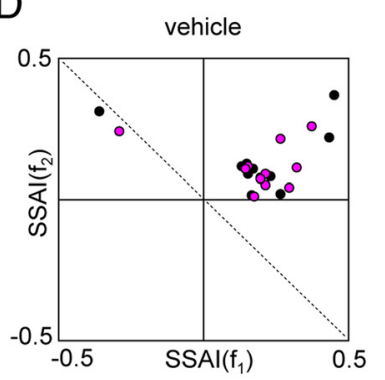

low MK801

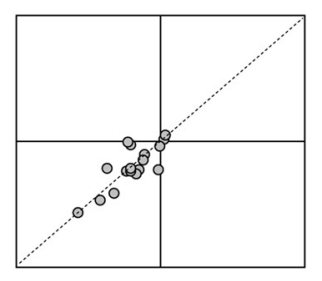

low MK801

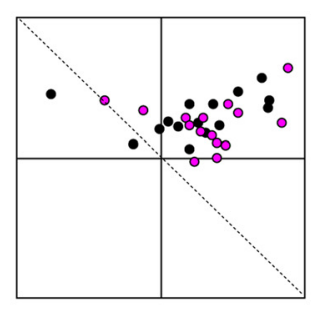

high MK801

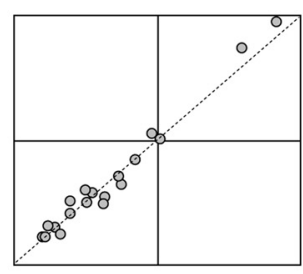

high MK801

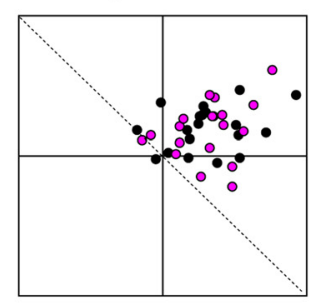

$\mathrm{B}$

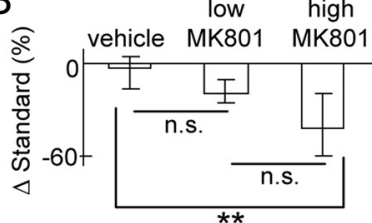

C

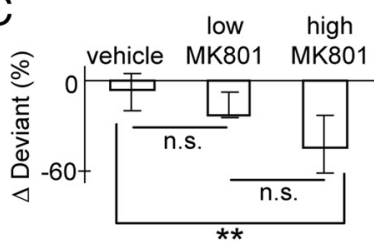

$E$

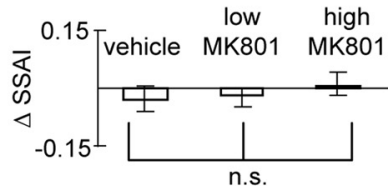

$\mathrm{F}$
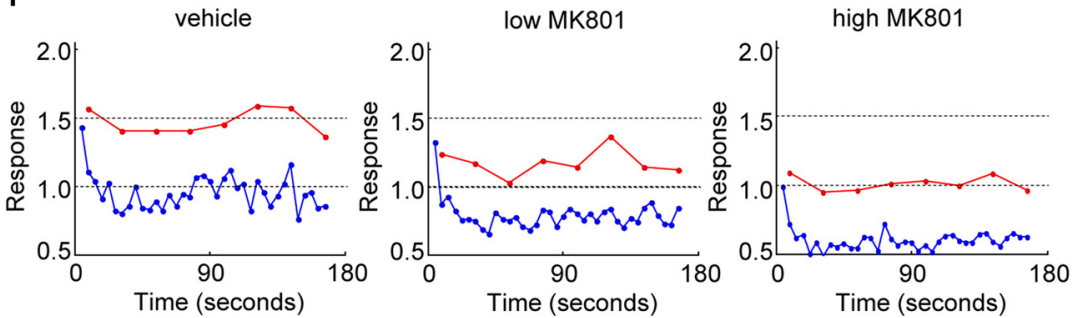

Figure 5. Effects of NMDA antagonist, MK801, on response magnitude and stimulus-specific adaptation for the auditory cortex multiunit population. $\boldsymbol{A}$, Percentage change in the magnitude of the response to the standard and to the deviant stimuli following injection of saline vehicle, low-dose MK801 (0.03 mg/kg, s.c.), or high-dose MK801 (0.1 mg/kg, s.c.). Each point comes from a multiunit site. $\boldsymbol{B}$, Median change, over the multiunit population, in the response to the standard following the treatment indicated. $\boldsymbol{C}$, Median change, over the population, in the response to the deviant following the treatment indicated. $\boldsymbol{D}$, The stimulus-specific adaptation indices for the $f_{1}$ or the $f_{2}$ stimuli, for individual multiunit sites, measured before (black) and after (magenta) the indicated treatment. $\boldsymbol{E}$, Median change, over the multiunit population, in the stimulus-specific adaptation index (averaged over the $f_{1}$ and $f_{2}$ stimuli) caused by each indicated treatment. $\boldsymbol{F}$, The temporal course of the population responses to the standard (blue) and deviant (red) during the frequency oddball paradigm following the treatment indicated. For each multiunit site, all postinjection responses are normalized to asymptote achieved by the vehicle standard; these normalized responses are then averaged across multiunit sites. Note that while MK801 injection causes a dose-dependent suppression of both standard and deviant responses relative to saline, the dynamics of adaptation for the standard and the deviant are unaffected. All error bars represent the upper and lower quartile values.

tential recordings from human studies. To increase confidence that LFPs were from the auditory cortex, only those electrodes where responsive multiunits were also recorded were analyzed. LFPs displayed a negativity with a latency of $\sim 20 \mathrm{~ms}$, which we term the N20 (see Materials and Methods). Like the multiunits, this N20 LFP component showed a significantly larger response to the deviant than standard tone in the frequency oddball paradigm (Fig. 6A, $C$; Sign test, $p<10^{-5}$ ). This is consistent with previous observations (Szymanski et al., 2009; von der Behrens et al., 2009). However, the N20 component of the LFP did not exhibit a stronger response to the deviant than to the control stimulus (Fig. 6B, $D$; Sign test, NS). These findings together indicated that, like the multiunit responses, the deviance-elicited increase in N20 was attributable to SSA, rather than to novelty. Although the average population LFP response also displayed an additional prominent positivity with a latency of $\sim 110 \mathrm{~ms}$, the existence of this component was more variable across recordings (see Materials and Methods). Additionally, we found that this "P110" component displayed no evidence for either SSA or for novelty (Fig. $6 A, B ; E, F$; Sign test, NS for deviant versus standard and deviant versus control).

Since the N20 component of the LFP displayed SSA in the oddball paradigm, we also analyzed its NMDA sensitivity. We found that, similar to the multiunit responses, the NMDA antagonist MK801 significantly depressed the N20 response to standard (Fig. $6 G$; Kruskal-Wallis ANOVA, $p<0.01$; post hoc Mann-Whitney: vehicle vs low-dose MK801, $p<0.05$, vehicle vs high-dose MK801, $p<0.05$, low-dose MK801 vs high-dose MK80, NS) as well as to deviant stimuli (Fig. 6 H; Kruskal-Wallis ANOVA, $p<0.01$; post hoc Mann-Whitney: vehicle vs low-dose MK801, $p<0.01$, vehicle vs high-dose MK801 $p<0.01$, low-dose MK801 vs high-dose MK801, NS). Indeed, despite the effects of MK801 on the N20 amplitude, there was no significant effect on SSA (Fig. 6I; Kruskal-Wallis ANOVA, NS, vehicle vs low-dose MK801 vs high-dose MK801). Thus the N20 component of the LFP displayed analogous characteristics to the multiunit responses, further establishing insensitivity of auditory cortex SSA to NMDA antagonists.

\section{Discussion}

\section{Properties encoded in auditory cortex units}

Whether auditory cortex units encode sensory novelty analogous to the human MMN, versus only SSA, has not been addressed adequately. Our auditory cortex multiunit dataset in the awake rat shows that these neurons respond more strongly to deviant than to standard stimuli in the frequency oddball paradigm, con- 
curring with previous studies (Ulanovsky et al., 2003; von der Behrens et al., 2009). While this property is consistent with that of a novelty-encoding signal, it can also be explained fully by a stimulus-specific adaptation process. Here we additionally tested auditory unit responses against several paradigms previously used in studies of the human MMN to differentiate between these possibilities (Näätänen et al., 2005).

First, a control condition added to the frequency oddball paradigm established a component in the human MMN which could not be explained by adaptation and is thought to encode novelty per se (Schröger and Wolff, 1996; Jacobsen and Schröger, 2001, 2003). A previous study of cat auditory cortex, using a similar control, has been interpreted to suggest that units in this area also encode novelty (Ulanovsky et al., 2003). However, unit responses were greater to deviant (i.e., "novel") than to control (i.e., "non-novel") stimuli only when the frequency difference between adjacent stimuli in the control condition (comprised of " 20 frequencies spanning approximately one octave", or a $\Delta f \sim 0.05$ between adjacent stimuli) was much smaller than the frequency difference between the standard and deviant in the oddball condition (i.e., this result held only for the $\Delta f=0.37$ oddball condition, but not the $\Delta f=0.10$ or $\Delta f=$ 0.05 conditions) (Ulanovsky et al., 2003). Since the amount of cross-frequency adaptation caused by neighboring tones on the response to a test tone increases as the frequency differences between the neighboring tones and the test tones decrease, more adaptation was likely present for their control tone than for the deviant tone in the $\Delta f=0.37$ oddball condition. Thus, while the increased deviant versus control response was interpreted to arise from a noveltyencoding component, the difference can also be explained by less adaptation to the deviant than to the control.

On the other hand, we used, similar to human studies, the same $\Delta f$ in both control and oddball conditions, which ensures that adaptation is not greater in the control condition (Jacobsen and Schröger, 2001). Contrasting to human MMN, we found that auditory cortex units failed to show a greater deviant than control response. Although this control condition has the possibility of underestimating novelty (Jacobsen and Schröger, 2001), we can conclude that under equivalent control conditions, rodent auditory cortex unit responses fail to show the novelty correlate established for the human MMN.

An additional means by which adaptation versus novelty components have been dissociated in neural signals is by the use of oddball paradigms focused on stimulus dimensions of intensity or duration. Both the human N1 and MMN ERP components are larger in response to loud deviant tones than to background soft tones; however, the MMN differentiates from the N1 by also responding more strongly to a soft deviant tone than to background loud tones (Näätänen and Picton, 1987; Näätänen et al., 1989). The latter property indicates that the MMN encodes intensity novelty per se, contrasting with the N1 response.
B
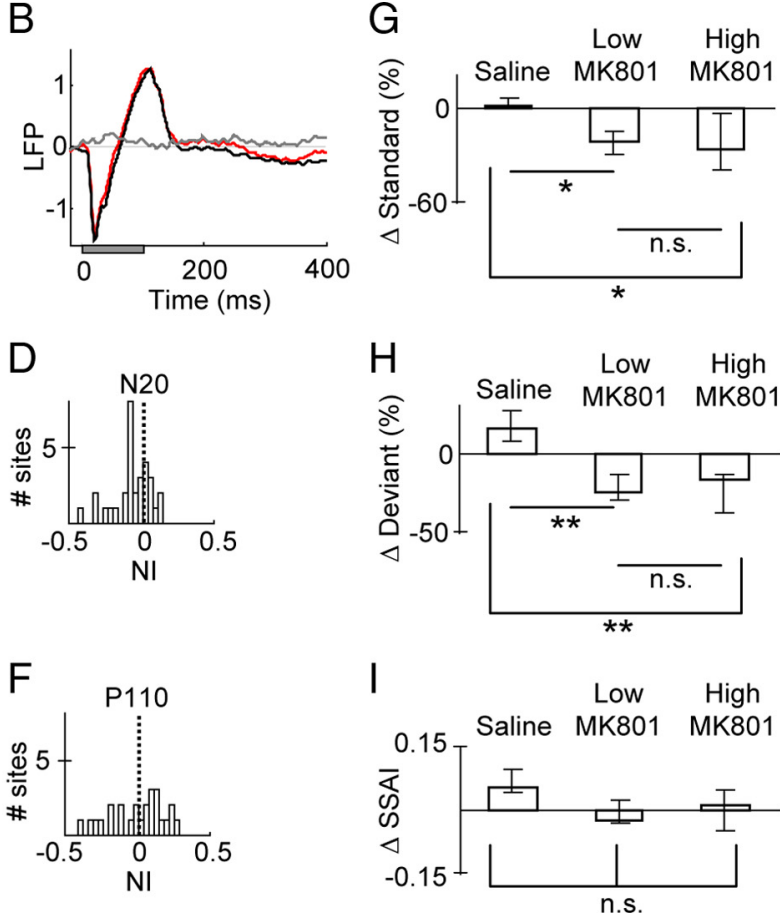

Figure 6. SSA but no novelty correlate in auditory cortex local field potential (LFP) responses. $\boldsymbol{A}$, Population evoked LFP in the frequency oddball paradigm averaged over all recording sites. The responses were normalized to the amplitude of

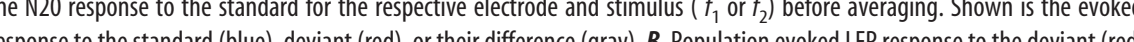

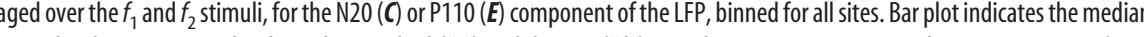
treatment indicated. $\boldsymbol{H}$, Median change, over the population, in the LFP N20 response to the deviant following the treatment indicated. I, Median change, over the population, in the LFP N20 stimulus-specific adaptation index, caused by the treatment indicated. All error bars represent the upper and lower quartile values.

Contrasting with the human MMN, we found that auditory cortex units respond more strongly to loud deviants than to loud standards, but not to soft deviants versus soft standards. Although a previous study concluded that cat auditory cortex units have an MMN-like response profile in an intensity oddball paradigm, that conclusion was based on pooling the results from loud and soft tones (Ulanovsky et al., 2003), a step which our data argue potentially confounds distinction of an adaptation from a novelty profile. Indeed, if raw data in that study are segregated for soft and loud stimuli separately, an effect similar to what we observed is evident; i.e., a large augmentation of the deviant vs standard for loud, but not soft tones (see Ulanovsky et al., 2003, their Fig. 4). The raw data from that study thus appear to concord with ours in failing to provide evidence for a novelty-encoding MMN-like process. Similarly, our duration oddball paradigm revealed no evidence that auditory cortex units encode duration novelty.

Rather, the data from the intensity and duration oddball paradigms can be more parsimoniously interpreted as reflecting a non-stimulus-specific gain adaptation process. The response elicited by a stimulus of a given intensity is smaller if it occurs against a background of repetitive loud (versus soft) tones; this background exists for soft tones occurring as deviants or loud tones occurring as standards. In the case of duration, the response elicited by a stimulus is smaller if it occurs against a background of repetitive long (versus short) tones.

Interestingly, recent studies from primate auditory cortex indicate that neurons with monotonic versus non-monotonic fir- 
ing rate-intensity functions show different forms of adaptation to stimulus intensity (Watkins and Barbour, 2008, 2010). Specifically, non-monotonic neurons maintain sensitivity to soft sounds in an environment where loud sounds predominated, a process which diverges from gain adaptation. These results suggest that monotonic and non-monotonic units may contribute differently to coding intensity, and could together code for novel soft and loud intensities. This idea remains to be tested in the oddball paradigm.

\section{Evaluating neural correlates of the human MMN}

It is worth considering why properties of primary auditory cortex neurons and human MMN might diverge, and reevaluating other neural signals as proposed MMN correlates. Although there is consensus that the auditory cortex contributes to the temporofrontal network generating MMN (Näätänen et al., 2007; Garrido et al., 2009), studies indicate that primary auditory cortex may generate the "sensory" (i.e., SSA) subcomponent of MMN, while a longer-latency "cognitive" (i.e., novelty) subcomponent might emanate from a partially distinct area including nonprimary auditory cortex (Opitz et al., 2005; Maess et al., 2007). Recent human studies have identified additional middle-latency evoked-potential components which responded to novelty per se (Grimm et al., 2010; Slabu et al., 2010), with latencies (30-40 $\mathrm{ms}$ ) aligning more closely with those of auditory cortex units. However, as with MMN, multiple areas are implicated as contributing to these potentials (Yvert et al., 2001). Future unit studies targeting additional auditory cortical areas might reveal responses aligning more closely with human MMN.

On the other hand, animal macroscopic LFP and EEG signals recorded during the oddball paradigm (for review, see Nelken and Ulanovsky, 2007) may share greater face-validity with the MMN than auditory units, being recorded by more analogous methods, and in some cases appearing as long-latency negative potentials. However, it is not known a priori what recording configuration in a given species, if any, would capture an MMN network. Thus, evaluation of these signals as putative MMN correlates, as with unit responses, should be based on whether they share important defining psychophysical (i.e., novelty-encoding) and pharmacological properties with the human MMN, as opposed to face-validity.

A series of studies of nonhuman primate demonstrated that LFP signals localized to supragranular layers of auditory cortex exhibit an NMDA-sensitive increased response to loud or soft oddball stimuli compared with standards (Javitt et al., 1992, 1994, 1996), suggesting they encode intensity-novelty. Further confidence in this signal as a general novelty- or MMN-correlate would be gained by testing it against stimulus dimensions in addition to intensity. On the other hand, our LFP recordings from rodent primary auditory cortex revealed SSA, but not novelty. The differences relative to the nonhuman primate studies could result from species differences, or relate to the different cortical layers targeted. Candidate EEG and LFP signals in other previous studies (reviewed in Nelken and Ulanovsky, 2007) were not tested against criteria distinguishing specifically between SSA and novelty; making this distinction will clarify the circuitry involved in the MMN and whether it is conserved across species.

\section{Pharmacology of SSA, MMN, and schizophrenia}

The observed disruption of the MMN in schizophrenia has generated great clinical interest, and motivated an effort to understand the mechanisms of sensory novelty detection. The disruption of MMN can be recapitulated in healthy volunteers by systemic NMDA antagonist administration, which also induces a host of other symptoms mimicking schizophrenia (Umbricht et al., 2000; Kreitschmann-Andermahr et al., 2001; Heekeren et al., 2008; but see Oranje et al., 2000). Understanding mechanisms by which NMDA antagonists disrupt MMN at a neuronal circuitlevel may provide a window into understanding the pathophysiology of schizophrenia.

The hypothetical convergence of SSA in auditory units as a component of MMN imparts obvious importance to delineating the sensitivity of SSA to NMDA antagonists: potentially disruption of SSA could explain NMDA antagonist sensitivity of the $\mathrm{MMN}$, as well as the dysfunction found in schizophrenia. Our investigation of this hypothesis, however, across a range of subanesthetic MK801 doses indicated a lack of specific effect on the magnitude or kinetics of SSA in auditory cortex unit responses. Thus the NMDA sensitivity reported for the MMN, and by extension the disruption of MMN observed in schizophrenia, may have a mechanistic locus outside of SSA, putatively involving the novelty detection component.

What are the biophysical and molecular mechanisms of SSA? Synaptic depression of thalamocortical or corticocortical synapses might be a major contributor. SSA, though existing in subcortical stations (Anderson et al., 2009; Malmierca et al., 2009) is stronger in cortex (Ulanovsky et al., 2003), especially beyond the thalamo-recipient layers (Szymanski et al., 2009). A synaptic depression process has been shown to mediate activity-dependent adaptation in olfactory (Best and Wilson, 2004) and somatosensory (Chung et al., 2002) cortices, and its properties are consistent with mediating the contextual dependence of auditory cortical neuronal responses in multiple paradigms (Ulanovsky et al., 2004; Asari and Zador, 2009; David et al., 2009). The potential molecular mechanisms of SSA to our knowledge have not been resolved in the auditory system, but our data argue that NMDA receptors do not contribute. On the other hand, evidence exists that transmitter depletion and presynaptic autoreceptor (mGluR II/III) activation contribute to sensory adaptation in the olfactory system (Best and Wilson, 2004); whether these mechanisms play a similar role supporting SSA in the auditory pathway is a testable hypothesis.

\section{References}

Anderson LA, Christianson GB, Linden JF (2009) Stimulus-specific adaptation occurs in the auditory thalamus. J Neurosci 29:7359-7363.

Asari H, Zador AM (2009) Long-lasting context dependence constrains neural encoding models in rodent auditory cortex. J Neurophysiol 102:2638-2656.

Barth DS, Di S (1990) Three-dimensional analysis of auditory-evoked potentials in rat neocortex. J Neurophysiol 64:1527-1536.

Best AR, Wilson DA (2004) Coordinate synaptic mechanisms contributing to olfactory cortical adaptation. J Neurosci 24:652-660.

Chung S, Li X, Nelson SB (2002) Short-term depression at thalamocortical synapses contributes to rapid adaptation of cortical sensory responses in vivo. Neuron 34:437-446.

David SV, Mesgarani N, Fritz JB, Shamma SA (2009) Rapid synaptic depression explains nonlinear modulation of spectro-temporal tuning in primary auditory cortex by natural stimuli. J Neurosci 29:3374-3386.

Garrido MI, Kilner JM, Stephan KE, Friston KJ (2009) The mismatch negativity: a review of underlying mechanisms. Clin Neurophysiol 120:453-463.

Grimm S, Escera C, Slabu L, Costa-Faidella J (2010) Electrophysiological evidence for the hierarchical organization of auditory change detection in the human brain. Psychophysiology. Advance online publication. Retrieved August 10, 2010. doi:10.1111/j.1469-8986.2010.01073.x.

Heekeren K, Daumann J, Neukirch A, Stock C, Kawohl W, Norra C, Waberski TD, Gouzoulis-Mayfrank E (2008) Mismatch negativity generation in the human 5HT2A agonist and NMDA antagonist model of psychosis. Psychopharmacology (Berl) 199:77-88. 
Hromádka T, Deweese MR, Zador AM (2008) Sparse representation of sounds in the unanesthetized auditory cortex. PLoS Biol 6:e16.

Jääskeläinen IP, Ahveninen J, Bonmassar G, Dale AM, Ilmoniemi RJ, Levänen S, Lin FH, May P, Melcher J, Stufflebeam S, Tiitinen H, Belliveau JW (2004) Human posterior auditory cortex gates novel sounds to consciousness. Proc Natl Acad Sci U S A 101:6809-6814.

Jacobsen T, Schröger E (2001) Is there pre-attentive memory-based comparison of pitch? Psychophysiology 38:723-727.

Jacobsen T, Schröger E (2003) Measuring duration mismatch negativity. Clin Neurophysiol 114:1133-1143.

Javitt DC, Schroeder CE, Steinschneider M, Arezzo JC, Vaughan HG Jr (1992) Demonstration of mismatch negativity in the monkey. Electroencephalogr Clin Neurophysiol 83:87-90.

Javitt DC, Steinschneider M, Schroeder CE, Vaughan HG Jr, Arezzo JC (1994) Detection of stimulus deviance within primate primary auditory cortex: intracortical mechanisms of mismatch negativity (MMN) generation. Brain Res 667:192-200.

Javitt DC, Steinschneider M, Schroeder CE, Arezzo JC (1996) Role of cortical $N$-methyl-D-aspartate receptors in auditory sensory memory and mismatch negativity generation: implications for schizophrenia. Proc Natl Acad Sci U S A 93:11962-11967.

Kreitschmann-Andermahr I, Rosburg T, Demme U, Gaser E, Nowak H, Sauer $\mathrm{H}$ (2001) Effect of ketamine on the neuromagnetic mismatch field in healthy humans. Brain Res Cogn Brain Res 12:109-116.

Maess B, Jacobsen T, Schröger E, Friederici AD (2007) Localizing preattentive auditory memory-based comparison: magnetic mismatch negativity to pitch change. Neuroimage 37:561-571.

Malmierca MS, Cristaudo S, Pérez-González D, Covey E (2009) Stimulusspecific adaptation in the inferior colliculus of the anesthetized rat. J Neurosci 29:5483-5493.

May PJ, Tiitinen H (2010) Mismatch negativity (MMN), the devianceelicited auditory deflection, explained. Psychophysiology 47:66-122.

Michie PT, Budd TW, Todd J, Rock D, Wichmann H, Box J, Jablensky AV (2000) Duration and frequency mismatch negativity in schizophrenia. Clin Neurophysiol 111:1054-1065.

Näätänen R, Picton T (1987) The N1 wave of the human electric and magnetic response to sound: a review and an analysis of the component structure. Psychophysiology 24:375-425.

Näätänen R, Paavilainen P, Alho K, Reinikainen K, Sams M (1989) Do event-related potentials reveal the mechanism of the auditory sensory memory in the human brain? Neurosci Lett 98:217-221.

Näätänen R, Jacobsen T, Winkler I (2005) Memory-based or afferent processes in mismatch negativity (MMN): a review of the evidence. Psychophysiology 42:25-32.

Näätänen R, Paavilainen P, Rinne T, Alho K (2007) The mismatch negativ- ity (MMN) in basic research of central auditory processing: a review. Clin Neurophysiol 118:2544-2590.

Nelken I, Ulanovsky N (2007) Mismatch negativity and stimulus-specific adaptation in animal models. J Psychophysiol 21:214-223.

Opitz B, Schröger E, von Cramon DY (2005) Sensory and cognitive mechanisms for preattentive change detection in auditory cortex. Eur J Neurosci 21:531-535.

Oranje B, van Berckel BN, Kemner C, van Ree JM, Kahn RS, Verbaten MN (2000) The effects of a sub-anaesthetic dose of ketamine on human selective attention. Neuropsychopharmacology 22:293-302.

Sams-Dodd F (1996) Phencyclidine-induced stereotyped behaviour and social isolation in rats: a possible animal model of schizophrenia. Behav Pharmacol 7:3-23.

Schröger E, Wolff C (1996) Mismatch response of the human brain to changes in sound location. Neuroreport 7:3005-3008.

Slabu L, Escera C, Grimm S, Costa-Faidella J (2010) Early change detection in humans as revealed by auditory brainstem and middle-latency evoked potentials. Eur J Neurosci. Advance online publication. Retrieved August 3, 2010. doi:10.1111/j.1460-9568.2010.07324.x.

Szymanski FD, Garcia-Lazaro JA, Schnupp JW (2009) Current source density profiles of stimulus-specific adaptation in rat auditory cortex. J Neurophysiol 102:1483-1490.

Ulanovsky N, Las L, Nelken I (2003) Processing of low-probability sounds by cortical neurons. Nat Neurosci 6:391-398.

Ulanovsky N, Las L, Farkas D, Nelken I (2004) Multiple time scales of adaptation in auditory cortex neurons. J Neurosci 24:10440-10453.

Umbricht D, Krljes S (2005) Mismatch negativity in schizophrenia: a metaanalysis. Schizophr Res 76:1-23.

Umbricht D, Schmid L, Koller R, Vollenweider FX, Hell D, Javitt DC (2000) Ketamine-induced deficits in auditory and visual context-dependent processing in healthy volunteers: implications for models of cognitive deficits in schizophrenia. Arch Gen Psychiatry 57:1139-1147.

von der Behrens W, Bäuerle P, Kössl M, Gaese BH (2009) Correlating stimulus-specific adaptation of cortical neurons and local field potentials in the awake rat. J Neurosci 29:13837-13849.

Watkins PV, Barbour DL (2008) Specialized neuronal adaptation for preserving input sensitivity. Nat Neurosci 11:1259-1261.

Watkins PV, Barbour DL (2010) Level-tuned neurons in primary auditory cortex adapt differently to loud versus soft sounds. Cereb Cortex. Advance online publication. Retrieved August 11, 2010. doi:10.1093/ cercor/bhq079.

Yvert B, Crouzeix A, Bertrand O, Seither-Preisler A, Pantev C (2001) Multiple supratemporal sources of magnetic and electric auditory evoked middle latency components in humans. Cereb Cortex 11:411-423. 